\title{
Real-time generation of representations for cognitive models
}

\author{
RANDY J. BROU \\ Navy Personnel Research, Studies, and Technology, Millington, Tennessee
}

AND

Stephanie M. Doane And Gary L. Bradshaw

Mississippi State University, Mississippi State, Mississippi

\begin{abstract}
Computerized models of human cognition and behavior have been used to help researchers refine their theories of cognitive processes. Two difficulties inherent in using these cognitive models are that building representations of to-be-modeled groups or individuals is (1) time-consuming and (2) prone to error when performed manually. The use of computerized tools to build representations for cognitive models could alleviate these problems. Such a set of tools has been developed and applied to the task of modeling individual aviation pilots performing flight maneuvers in real time. A study conducted with 27 pilots confirms the accuracy of the computer-generated representations.
\end{abstract}

Computational cognitive modeling provides researchers with a powerful way to test and refine their theories (Gray, 2007). Although very useful, developing computational cognitive models is a challenging process. This challenge exists in part because the models must contain accurate representations of the to-be-modeled groups or individuals. In the case of modeling individuals, this means that each individual's knowledge base and relevant characteristics (e.g., working memory capacity) must be uniquely constructed. Determining the contents of a knowledge base or the value for an individual parameter can be time-consuming and error prone if performed manually (Jodlowski, Carruth, Lowe, \& Doane, 2003). Using computerized tools offers two advantages to researchers building representations for cognitive models: time savings and removal of human error. The current research represents an effort to build and validate a set of computerized tools that automate the processes of building accurate representations for a cognitive model. Specifically, the tools have been applied to the task of building representations for ADAPT, a computational cognitive model of individual aviation pilots' action planning (Doane \& Sohn, 2000).

In previous research, Doane and colleagues demonstrated the capability of ADAPT to predict individual aviation pilots' eye fixations and control movements during simulated flight tasks (Doane \& Sohn, 2000; Sohn $\&$ Doane, 2002). ADAPT accounted for over $80 \%$ of the variance in the sequence-dependent execution of individual pilot actions when used to simulate the performance of 25 human pilots on seven flight maneuvers. The rep- resentations for each of the individual pilots modeled by ADAPT were carefully constructed by hand. This was accomplished in part by having trained raters apply contextsensitive scoring rules to small portions of observed flight performance data and deciding what knowledge individual pilots possessed. This scoring effort typically lasted several hours per pilot. So, although the results of the original effort were impressive, the usefulness of the ADAPT model as an applied tool was limited because of the time necessary to generate individual pilot representations. As part of an effort to transition ADAPT into an applied tutoring technology for novice pilots, a set of computerized tools was developed to automate the process of building individual representations. In the following sections, a brief description of the parts of the ADAPT model will be provided. Following the description of ADAPT, the results of an effort to validate the quality of the representations generated by the computerized tools will be described.
ADAPT
Cognitive models are developed within larger frame- works known as cognitive architectures. Cognitive archi- tectures are general theories about the nature of informa- tion processing that account for performance across a wide range of tasks (Byrne, 2003; Newell, 1990). Some of the better known cognitive architectures include ACT-R and Soar (e.g., Anderson et al., 2004; Rosenbloom, Laird, Newell, \& McCarl, 2002). Each cognitive architecture makes certain assumptions about how cognitive tasks are 
performed. The ADAPT model is based on a cognitive architecture known as the construction-integration $(C-I)$ theory (Kintsch, 1988). The major theoretical premise of $\mathrm{C}-\mathrm{I}$ is that comprehension-based mechanisms identical to those used to understand a list of words, narrative prose, and algebraic word problems constrain problem-solving episodes as well.

The construction-integration theory was initially developed to explain phenomena, such as word sense disambiguation, common in text comprehension. The theory describes how context helps listeners or readers find appropriate meaning for words that have multiple meanings; for example, if a person is discussing paychecks and hears the word "bank," he or she will probably interpret the word as referring to a building rather than to the land near a body of water. Kintsch's (1988) theory shows how such decisions can be explained by representing memory as an associative network with nodes containing propositional representations of knowledge about the current state of the world (context-dependent), general (context-independent) declarative facts, and if/then rules that represent possible plans of action (Mannes \& Kintsch, 1991). In C-I, a set of possible interpretations of an ambiguous word is first constructed by allowing a set of weak symbolic production rules to spread "activation" through an associative network on the basis of superficial similarities between propositional representations of knowledge. After the initial spread of activation, the associated network of knowledge is integrated via a constraint-satisfaction algorithm. This algorithm propagates activation throughout the network, strengthening connections between items relevant to the current task context and inhibiting or nullifying connections between irrelevant items. Each decision about what meaning to assign to a word (or about what action to take next in the case of the ADAPT model) is referred to as a C-I cycle.

Like all C-I models, ADAPT represents the three classes of knowledge proposed by Kintsch $(1988,1998)$ : world knowledge, general knowledge, and plan element knowledge. In ADAPT, each class of knowledge is limited to aviation-specific information, but the knowledge included in another C-I model could represent any other domain. Furthermore, ADAPT represents individual pilot characteristics, such as working memory capacity. Each part of the ADAPT representation will be discussed in turn.

\section{World Knowledge}

World knowledge represents information about the current state of a task environment. In ADAPT, a pilot can possess knowledge about the current and desired states of an airplane, or the relationship between these values (e.g., altitude is higher than desired value). World knowledge can also include information about the current goals of a flight maneuver. World knowledge is contextually sensitive and fluid; that is, it changes with the state of the world throughout the simulated flight performance.

\section{General Knowledge}

General knowledge refers to factual information. In ADAPT, general knowledge can include facts about flying an aircraft, such as the relationship between control input and plane performance, as well as knowledge of flight dynamics, display instruments, and control movements.

\section{Plan Element Knowledge}

Plan element knowledge represents "executable" (procedural) knowledge. Plan elements describe actions and the conditions under which they can be taken; that is, they describe condition-action rules that can be executed if conditions "in the world" (i.e., in the current task context) match those specified in the plan element. Plan elements consist of three parts: name, preconditions, and outcomes. The name describes the action (e.g., "look at the altimeter"). The preconditions refer to world knowledge or general knowledge that must exist before a plan element can be executed. For example, a plan element for looking at the altimeter might not be able to fire unless the pilot's world knowledge reflects that he/she does not know the plane's current altitude. Plan element outcomes contain propositions added to, or updating, the model's world knowledge when the plan element is fired; for example, once the pilot has looked at the altimeter, the world knowledge will change to reflect the fact that the pilot knows the plane's current altitude.

\section{ADAPT Memory Constraints}

In addition to representing the "in-the-world," general, and plan element knowledge of individual pilots, ADAPT included parameters describing the working memory differences that exist among the pilots. Working memory was used as part of the model, because it serves an important function for complex task performance in a dynamically changing environment (e.g., Durso \& Gronlund, 1999; Sohn \& Doane, 2003). To account for the impact of working memory limitations on piloting performance, two memory components were incorporated in the ADAPT model to represent capacity and decay constraints.

Capacity function. Pilots, like other task performers, can only retain a limited amount of information at one time. Thus, in ADAPT, the number of "in-the-world" propositions accessible to the model was restricted. When the number of "in-the-world" propositions exceeded working memory capacity limitations (represented as a parameter value), ADAPT began to delete propositions, starting with those having the lowest activation. (For a detailed discussion of how activation is calculated, see Doane \& Sohn, 2000). For example, if the capacity limits were set to four propositions, the "in-the-world" propositions that were not among the fourth most activated were deleted. This procedure simulated context-sensitive working memory limitations, because proposition activation was constrained by relevance to the current task context. This capacity function was applied only to the "in-theworld" propositions.

Decay function. ADAPT also incorporated a decay component. In the dynamic context of flight, world information must be updated in a timely manner. Decay was represented by tracking the age of each proposition "in the world," where propositional age increased by one after each $\mathrm{C}-\mathrm{I}$ cycle. A decay threshold was used to automati- 
cally delete old propositions following each $\mathrm{C}-\mathrm{I}$ cycle. For example, if the decay threshold was set at 7, "in-the-world" propositions older than seven C-I cycles were deleted.

\section{Constructing Individual Representations}

The goal of developing ADAPT was to model individual pilots; in order to do so, a knowledge base and memory parameters for each individual had to be generated. Originally, representations were generated by having trained raters apply explicit scoring rules by hand. For example, one rule might state that if a pilot manipulated the elevator and looked at the altimeter in parallel, the knowledge base for that pilot's model should include knowledge that the elevator control is used to change altitude and that the altimeter indicates altitude. In addition, the knowledge base should include a plan element for changing aircraft altitude that included manipulation of the elevator and fixation on the altimeter. Working memory parameters could likewise be generated by hand by evaluating the time between subsequent fixations on instruments. Once again, detailed descriptions of the rules used can be found in Doane and Sohn (2000).

As mentioned earlier, the representation-building process was very time consuming and prone to human error, requiring multiple inspections of the representations to ensure that they were accurately generated. In the present research, a set of computerized tools was developed to automatically generate the knowledge base and memory parameters of individual pilots. These tools were developed to take as input information about the pilot's current situation (e.g., current altitude and rate of climb), eye fixations, and control movements. Given those data as input, the tools evaluated the pilot's actions in the context of ongoing flight situations and generated a representation of the pilot in the form of a knowledge base and memory characteristics.

As an example of the computerized tools working, consider the following case. A pilot flying straight and level looks at the heading indicator and notes that the plane is headed due south. Having been given instructions to turn $180^{\circ}$ and fly north, the pilot applies right pressure to the aileron and begins to bank. At a rate of 60 times/sec, the computerized tools are constantly monitoring the extent of the pilot's professional knowledge. In this case, the tools first register the fact that the pilot's current heading and desired heading do not match. Next, they register the fact that the pilot begins a fixation on the heading indicator, which by itself is not enough to credit the pilot with any knowledge, but when the aileron is manipulated by the pilot, the tools take note of this as well because this set of behaviors has triggered a rule: The pilot sees an incorrect heading and manipulates the aileron in response. Thus, the tools score the pilot for knowing the association between manipulating the aileron and changing heading. The rules are context sensitive in that the pilot would not have been credited with the knowledge had the same actions been performed when the plane's heading was correct. Additionally, in some cases a pilot may not be credited by the tools for knowledge without demonstrating certain behaviors multiple times within the correct context; this reduces the likelihood of giving pilots credit for knowledge when they only perform a set of actions by chance. As illustrated in this example, the tools performed the same functions as a trained rater would, but applied the context-sensitive scoring rules in real time (i.e., while the pilot completed the flight maneuvers).

What follows is the description of a study conducted to validate the quality of the representations generated by the computerized tools in real time. The ADAPT model has been shown to accurately predict sequence-dependent pilot behaviors when provided with accurate representations of individual pilots (Doane \& Sohn, 2000). Thus, the true test of the computerized tools is whether or not they can provide the ADAPT model with individual pilot representations of sufficient quality to maintain the model's previously displayed predictive power. For the purposes of this test, quality of representations will be operationally defined as the quality of the model's prediction of the individual pilots' behaviors.

\section{METHOD}

\section{Participants}

Twenty-seven pilots were recruited from the flight school at Delta State University. Each pilot was paid $\$ 25 / \mathrm{h}$ to participate in the $2-\mathrm{h}$ experiment. To provide information on their expertise, each pilot completed a preexperimental questionnaire that consisted of questions about flight time, instrument time, number of aircraft flown, number of certificates earned, and flight time in the previous 90 days. Responses to the questionnaire items were submitted to a principal-components factor analysis (Varimax rotation). The resulting factors were used to classify pilots into groups on the basis of experience. The analysis provided two factors with eigenvalues greater than 1 , accounting for $77.6 \%$ of the variance in the data. The first factor, labeled Flight Training, accounted for $63.3 \%$ of the variance. This factor included questions about total flight hours, number of certificates obtained, number of courses taken, number of different types of aircraft flown, and total instrument hours. The second factor, labeled Simulation and Recent Experience, accounted for 14.3\% of the variance. This factor included questions about total simulated flight hours and instrument flight time in the last 90 days.

A discriminant analysis based on the two previously mentioned factors was used to classify pilots into different levels of expertise. The pilots were classified into three classes: novice, intermediate, and expert, containing nine pilots each.

\section{Apparatus}

Flight simulator. A Dell Precision 340 computer with a highend Nvidia GeForce graphics card executed the flight simulator experiment. A 21-in. color monitor displayed the instrument panel rendered by Microsoft Flight Simulator. Figure 1 shows a screenshot of the Microsoft Flight Simulator 2000 software displaying a simplified instrument panel with the standard primary instruments: airspeed indicator, attitude indicator, altimeter, turn coordinator, heading indicator, vertical speed indicator (VSI), and tachometer; a cockpit instruction panel; an overhead map of the flight path; and a timer. The cockpit instruction panel consists of three boxes that indicate the starting state of the aircraft ("Start" box), the maneuvers the pilot was required to execute in the current segment ("Change to" box), and the goal state of the aircraft for the current segment. The "Start" box specified the heading, altitude, and airspeed that the aircraft should maintain for the first $30 \mathrm{sec}$ of the segment. The "Change to" box specified the flight maneuver (e.g., "Increase airspeed to 100 knots") and the amount of time allotted to the maneuver. The "Finish" box displayed the desired heading, altitude, and airspeed of the aircraft at the end of the maneuver. Pilots were 


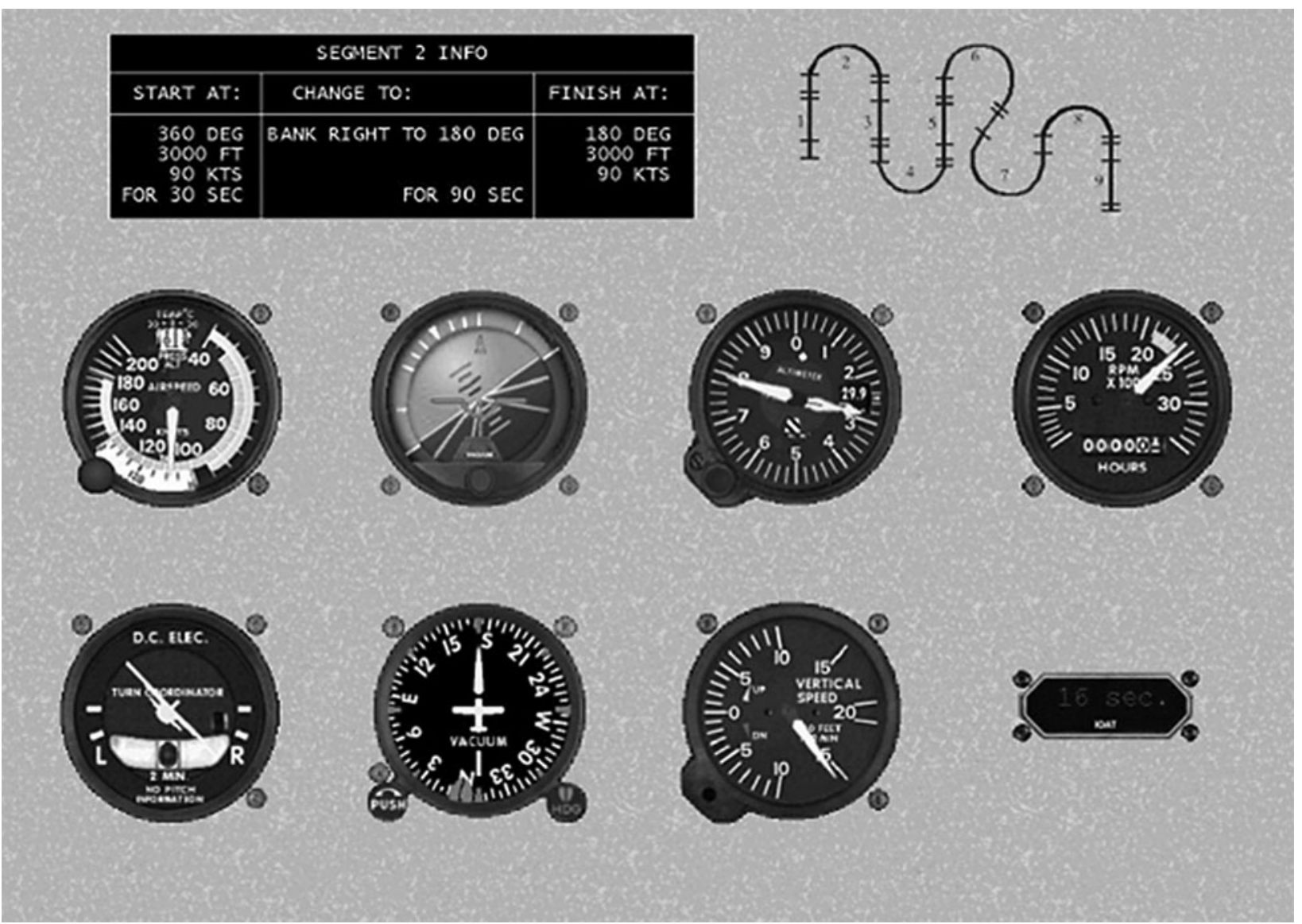

Figure 1. Example of a flight simulator cockpit instrument display.

expected to bring the aircraft within $\pm 50 \mathrm{ft}(15.24 \mathrm{~m})$ of desired altitude, $\pm 5^{\circ}$ of desired heading, and \pm 5 knots of desired airspeed before the end of each segment. Pilots used a realistic Cirrus yokeand-throttle control on the simulated aircraft. The yoke-and-throttle control was placed beneath the monitor. The flight status of the aircraft and the pilot's control movements were recorded throughout each segment of simulated flight.

Head-mounted eyetracker. An Applied Sciences Laboratory Series 5000 head-mounted eyetracker recorded eye scan measures. Sampling and output rate of the tracking camera was $60 \mathrm{~Hz}$. Pilot fixations and dwell times were time synched with the flight status and control movement data and were recorded.

\section{Procedure}

Pilots arriving for the experiment read and signed a consent form and completed the previously mentioned questionnaire. They were then instructed to read instructions regarding the layout of the instrument panel and yoke-and-throttle controls. The experiments consisted of three parts: familiarization flight, practice flight, and recorded flight. Pilots were required to take a few minutes' break between each part of the session. Overall, the experiment lasted approximately $2 \mathrm{~h}$, and was completed in one sitting.

Familiarization flight. After reading the instructions, the experimenter launched Microsoft Flight Simulator in an untimed "free flight" mode. The flight began at an altitude of $3,000 \mathrm{ft}$, a heading of $360^{\circ}$, and an airspeed of 100 knots $(\mathrm{kn})$. The experimenter verbally directed the pilots through a series of maneuvers to familiarize them with the control dynamics of the simulation environment without the pressure of a time constraint. The experimenter observed the pilots' completing each maneuver. When pilots were within bounds
( \pm 50 feet $[15.24 \mathrm{~m}]$ for altitude, \pm 5 knots for airspeed, and $\pm 5^{\circ}$ for heading) - they would be directed to perform the next maneuver. After completing the maneuvers, the pilots spent an additional 5-15 min experimenting with the simulation without experimenter direction.

Practice flight. After the familiarization flight, pilots practiced flying seven different flight segments that required airspeed, altitude, and/or heading changes. The flight segments required pilots to perform basic flight maneuvers manipulating the altitude, airspeed, and/or heading of the aircraft. A 30-sec straight and level lead-in leg maintained by an autopilot mechanism preceded each segment. This provided the pilots with time to read the instructions for the upcoming segment. The maneuvers required for each flight segment were displayed in the cockpit instruction panel. Pilots were allotted $90 \mathrm{sec}$ to accomplish the maneuver in the first six segments and $115 \mathrm{sec}$ in the seventh segment. Whether or not the participants were within bounds at the end of the allotted period, the computer ended the segment and the simulated aircraft was placed at the beginning of the next lead-in leg. This procedure enabled the recording of independent segments of flight performance for each pilot.

Flight segments varied in complexity. The first three segments of the simulation required a change in only one flight axis: airspeed, heading, or altitude (the other two axes were to be maintained at starting values). The fourth through sixth segments required changes in two flight axes, and the seventh segment required a change in all three axes. The time required to complete all seven segments was approximately $15 \mathrm{~min}$.

Recorded flight. After a short break, the experimenter calibrated the pilots' head-mounted eyetrackers. Once this was done, the experimenter launched the same seven-segment series from the practice 
flight. Pilots completed the maneuvers and their performance was recorded. Recorded data from the first three flight segments were sent to the automated representation-building tools in real time. These data included the eye movements and control manipulations pilots made, as well as the status of the aircraft (e.g., current altitude, rate of climb, etc.) at a $60-\mathrm{Hz}$ rate of sampling. On the basis of the data from these segments, the tools generated individual pilot representations that were then used in ADAPT models to predict flight performance on all seven flight segments. Following completion of the last segment, pilots were given a debriefing sheet and were paid for their time.

\section{RESULTS}

As an initial check of the computerized tools, representations generated by hand were compared with the output of the tools to determine whether any of the hand-scored knowledge elements were missed. Although no hand-scored knowledge elements were missing from the computergenerated representations, there were several instances in which the computerized tools scored additional knowledge elements. Upon closer examination, those additional knowledge elements represented instances of oversight on the part of the human rater. This effectively demonstrated attainment of the goal of reducing human error through automation.

In order to further validate the quality of the representations generated by the computerized tools (as defined by ADAPT's ability to predict pilot behaviors), a fit between individual pilot and model behaviors was calculated. To quantify this fit, the match between the sequence of actions observed for human pilots and the sequence of corresponding plan elements fired by their ADAPT models was calculated. The match between human and modeled pilot behaviors was calculated only for comparable flight situations; that is, the status of the actual and modeled flight situation matched at the beginning of each segment, but if the model executed a plan element that the human pilot did not, the modeled and actual flight situations could diverge. Mismatches of behavior due to dissimilar flight situations were not of interest in determining the fit.

The specific method of calculating a fit between pilot and model behaviors consisted of the following five steps.

1. Identify the beginning and ending of each comparable flight situation within each segment of flight.

2. List each action taken by the pilot and each taken by the model in sequential order during each comparable flight situation (a list of the possible actions the models could perform is given in the Appendix).

3. Compare the sequence of pilot behaviors and model behaviors within a flight situation in order to identify which actions occurred in both sequences.

4. Calculate for each flight situation the number of matching behaviors divided by the number of total behaviors exhibited by the model (i.e., if the model in a given situation performed six actions, of which the human pilot performed five, the match would be equal to 5 divided by 6 , or $83.3 \%$ for that situation).

5. Average the matches for each comparable flight situation (over all flight segments, or over flight segments of similar complexity) to obtain reported matches.

The overall mean percent matches for the sequence of behaviors were $84 \%, 86 \%$, and $83 \%$ for the novice, inter-
Table 1

Mean Percent Match and Standard Error for Action Plans Between Models and Pilots As a Function of Flight Maneuver Complexity and Expertise

\begin{tabular}{|c|c|c|c|c|c|c|}
\hline \multirow[b]{2}{*}{ Complexity } & \multicolumn{2}{|c|}{ Novice } & \multicolumn{2}{|c|}{ Intermediate } & \multicolumn{2}{|c|}{ Expert } \\
\hline & $M$ & $S E$ & $M$ & $S E$ & $M$ & $S E$ \\
\hline Single axis & 85.44 & 2.57 & 88.01 & 1.95 & 88.06 & 1.91 \\
\hline Double axis & 83.57 & 2.17 & 83.23 & 1.95 & 79.21 & 2.55 \\
\hline Triple axis & 82.59 & 3.38 & 86.10 & 2.53 & 80.65 & 3.50 \\
\hline
\end{tabular}

Table 2

Mean Percent Match and Standard Error for Action

Plans Between Models and Pilots As a Function of Flight Maneuver Status and Expertise

\begin{tabular}{|c|c|c|c|c|c|c|}
\hline \multirow[b]{2}{*}{ Status } & \multicolumn{2}{|c|}{ Novice } & \multicolumn{2}{|c|}{ Intermediate } & \multicolumn{2}{|c|}{ Expert } \\
\hline & $M$ & $S E$ & $M$ & $S E$ & $M$ & $S E$ \\
\hline Initiating & 83.30 & 2.58 & 89.92 & 1.64 & 88.41 & 1.97 \\
\hline Maintaining & 87.33 & 3.25 & 82.33 & 2.59 & 82.21 & 2.93 \\
\hline Finishing & 81.98 & 2.24 & 83.89 & 2.08 & 77.56 & 2.84 \\
\hline
\end{tabular}

Table 3

Mean Proportion of Human Flight Time Appropriate for Calculating Matches Between Models and Pilots As a Function of Flight Maneuver Complexity and Expertise

\begin{tabular}{|c|c|c|c|c|c|c|}
\hline \multirow[b]{2}{*}{ Complexity } & \multicolumn{2}{|c|}{ Novice } & \multicolumn{2}{|c|}{ Intermediate } & \multicolumn{2}{|c|}{ Expert } \\
\hline & $M$ & $S E$ & $M$ & $S E$ & $M$ & $S E$ \\
\hline Single axis & .37 & .05 & .40 & .04 & .39 & .07 \\
\hline Double axis & .41 & .03 & .48 & .01 & .48 & .01 \\
\hline Triple axis & .44 & .01 & .45 & .01 & .35 & .01 \\
\hline
\end{tabular}

mediate, and expert pilots, respectively. Table 1 shows a breakdown of the matches by flight maneuver complexity (i.e., changes required on one, two, or three flight axes) and expertise. Table 2 shows a breakdown of the matches by flight maneuver status (i.e., initiating a change, maintaining a change, or finishing a change) and expertise. Table 3 shows the proportion of human flight time that the human and model pilots spent in similar flight situations for which it was appropriate to calculate a match. In all cases, the representations generated by the computerized tools were sufficient to allow for high matches of individual performance. Note that there are no significant differences in the matches between pilot and model behaviors as a function of expertise $\left[F(2,21)=0.62, M S_{\mathrm{e}}=0.02, p>.54\right]$, complexity $\left[F(2,42)=0.43, M S_{\mathrm{e}}=0.01, p>.65\right]$, or flight status $\left[F(2,42)=0.05, M S_{\mathrm{e}}=0.01, p>.94\right]$.

\section{DISCUSSION}

The high percentage of matching behaviors between human and model pilots indicates that the computergenerated representations are sufficiently accurate. Given that there were no significant differences between matches generated for experts, intermediates, and novices, it appears that the tools are capable of generating accurate representations across expertise groups. Moreover, the representations are capturing the necessary elements of pilot knowledge to account for performance in a wide range of flight maneuvers and the various stages of executing those 
maneuvers. Additionally, the amount of time the pilots and models were in similar flight situations was similar to that originally reported by Doane and Sohn (2000), a further indication that computerized tools have successfully replicated the function of trained human raters.

Computerized tools for building representations have the obvious advantage of being faster and less error prone than humans. Additional advantages may also be seen if the representations need to be updated in real time, as task performers learn new facts or procedures. This opens the door for employing cognitive models as tutors in dynamic task performance. Although the tools described in this study have been applied to building representations for one specific model, they are designed to be more generic and could be modified for use with other models. New research technologies, such as these tools, have a great potential to help us advance our understanding of human cognition, particularly when combined with such powerful methods as cognitive modeling.

\section{AUTHOR NOTE}

The authors gratefully acknowledge the support of the Office of Naval Research, Warfighter Performance Division (Code 34) in the form of Grant ONR N000140210152, awarded to S.M.D. Correspondence concerning this article should be addressed to S. M. Doane, Department of Psychology, P.O. Box 6161, Mississippi State University, MS 39762 (e-mail: smd39@msstate.edu).

\section{REFERENCES}

Anderson, J. R., Bothell, D., Byrne, M., Douglass, S., Lebiere, C., \& QIN, Y. (2004). An integrated theory of the mind. Psychological Review, 111, 1036-1060.

Byrne, M. D. (2003). Cognitive architecture. In J. Jacko \& A. Sears (Eds.), The human-computer interaction handbook: Fundamentals, evolving technologies, and emerging applications (pp. 95-114). Hillsdale, NJ: Erlbaum.

DoAne, S. M., \& Sohn, Y. W. (2000). ADAPT: A predictive cognitive model of user visual attention and action planning. User Modeling \& User Adapted Interaction, 10, 1-45.

Durso, F. T., \& Gronlund, S. D. (1999). Situation awareness. In F. T. Durso, R. Nickerson, R. Schvaneveldt, S. Dumais, S. Lindsay, \& M. Chi (Eds.), The handbook of applied cognition (pp. 283-314). New York: Wiley.

GraY, W. D. (2007). Integrated models of cognitive systems. New York: Oxford University Press.
Jodlowski, M. T., Carruth, D. W., Lowe, D., \& Doane, S. M. (2003, May). Knowledge scoring engine (KSE) for real-time knowledge base generation. In Proceedings of the 12th Conference on Behavior Representation in Modeling and Simulation (pp. 435-437). Scottsdale, AZ.

KINTSCH, W. (1988). The use of knowledge in discourse processing: A construction-integration model. Psychological Review, 95, 163-182.

KINTSCH, W. (1998). Comprehension: A paradigm for cognition. New York: Cambridge University Press.

Mannes, S. M., \& Kintsch, W. (1991). Routine computing tasks: Planning as understanding. Cognitive Science, 15, 305-342.

Newell, A. (1990). Unified theories of cognition. Cambridge, MA Harvard University Press.

Rosenbloom, P. S., LAird, J. E., Newell, A., \& McCARL, R. (2002). A preliminary analysis of the SOAR architecture as a basis for general intelligence. Artificial Intelligence, 47, 289-325.

SoHn, Y. W., \& DoAne, S. M. (2002). Evaluating comprehension-based user models: Predicting individual user planning and action. User Modeling \& User Adapted Interaction, 12, 171-205.

SoHn, Y. W., \& DoAne, S. M. (2003). Roles of working memory capacity and long-term working memory skill in complex task performance. Memory \& Cognition, 31, 458-466.

\section{APPENDIX \\ Actions the ADAPT Model Could Perform}

Look at airspeed indicator

Look at attitude indicator

Look at altimeter

Look at turn coordinator

Look at heading indicator

Look at vertical speed indicator

Look at tachometer

Pull throttle back

Push throttle forward

Pull elevator back

Push elevator forward

Apply left pressure to aileron

Apply right pressure to aileron

Note-The length of fixations on a particular instrument was not represented in the model, nor was the length of control movements. There were, however, different plan elements representing "large" or "small" control movements. For further discussion on these matters, see Doane and Sohn (2000).

(Manuscript received December 6, 2007; revision accepted for publication June 24, 2008.) 